\title{
GIANO-TNG spectroscopy of red supergiants in the young star cluster RSGC2 ${ }^{\star}$
}

\author{
L. Origlia ${ }^{1}$, E. Oliva ${ }^{2}$, R. Maiolino ${ }^{3}$, A. Mucciarelli ${ }^{4}$, C. Baffa ${ }^{2}$, V. Biliotti ${ }^{2}$, P. Bruno ${ }^{5}$, G. Falcini ${ }^{2}$, V. Gavriousev ${ }^{2}$,

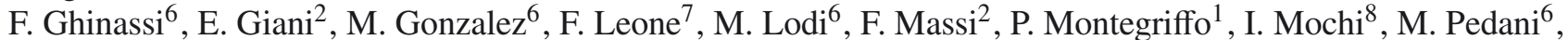 \\ E. Rossetti ${ }^{4}$, S. Scuderi ${ }^{5}$, M. Sozzi ${ }^{2}$, and A. Tozzi ${ }^{2}$
}

\author{
1 INAF - Osservatorio Astronomico di Bologna, via Ranzani 1, 40127 Bologna, Italy \\ e-mail: livia.origlia@oabo.inaf.it \\ 2 INAF - Osservatorio Astrofisico di Arcetri, Largo E. Fermi 5, 50125 Firenze, Italy \\ 3 University of Cambridge, Cavendish Lab., JJ Thomson Av., Cambridge CB3 OHE, UK \\ ${ }^{4}$ University of Bologna, Physics \& Astronomy Dept., Viale Berti Pichat 6-2, 40127 Bologna, Italy \\ 5 INAF - Osservatorio Astrofisico di Catania, via S. Sofia 78, 95123 Catania, Italy \\ ${ }^{6}$ INAF - TNG, ORM Astronomical Observatory, 38787 Garafia, TF, Spain \\ 7 University of Catania, Physics \& Astronomy Dept. via S. Sofia 78, 95123 Catania, Italy \\ ${ }^{8}$ Lawrence Berkeley National Laboratory, 1 Cyclotron Road, MS 2-400, Berkeley CA 94720, USA
}

Received 2 September 2013 / Accepted 4 November 2013

\section{ABSTRACT}

\begin{abstract}
Aims. The inner disk of the Galaxy has a number of young star clusters dominated by red supergiants that are heavily obscured by dust extinction and observable only at infrared wavelengths. These clusters are important tracers of the recent star formation and chemical enrichment history in the inner Galaxy.

Methods. During the technical commissioning and as a first science verification of the GIANO spectrograph at the Telescopio Nazionale Galileo, we secured high-resolution $(R \simeq 50000)$ near-infrared spectra of three red supergiants in the young Scutum cluster RSGC2.

Results. Taking advantage of the full YJHK spectral coverage of GIANO in a single exposure, we were able to identify several tens of atomic and molecular lines suitable for chemical abundance determinations. By means of spectral synthesis and line equivalent width measurements, we obtained abundances of $\mathrm{Fe}$ and other iron-peak elements such as $\mathrm{V}, \mathrm{Cr}, \mathrm{Ni}$, of alpha $(\mathrm{O}, \mathrm{Mg}, \mathrm{Si}, \mathrm{Ca}$ and $\mathrm{Ti})$ and other light elements (C, N, Na, Al, K, Sc), and of some s-process elements (Y, Sr). We found iron abundances between half and one third solar and solar-scaled $[\mathrm{X} / \mathrm{Fe}]$ abundance patterns of iron-peak, alpha and most of the light elements, consistent with a thin-disk chemistry. We found a depletion of $[\mathrm{C} / \mathrm{Fe}]$ and enhancement of $[\mathrm{N} / \mathrm{Fe}]$, consistent with $\mathrm{CN}$ burning, and low ${ }^{12} \mathrm{C} /{ }^{13} \mathrm{C}$ abundance ratios (between 9 and 11), requiring extra-mixing processes in the stellar interiors during the post-main-sequence evolution. Finally, we found a slight $[\mathrm{Sr} / \mathrm{Fe}]$ enhancement and a slight $[\mathrm{Y} / \mathrm{Fe}]$ depletion (by a factor of $\leq 2$ ), with respect to solar.
\end{abstract}

Key words. techniques: spectroscopic - supergiants - stars: abundances - infrared: stars

\section{Introduction}

High-resolution spectroscopy in the near-infrared (NIR) is a powerful tool for measuring chemical abundances of cool giant and supergiant stars. It is especially critical in very reddened environments such as the inner Galaxy, where extinction is so severe as to prevent any reliable measurement at shorter wavelengths.

Recently, our team made the first technical commissioning of GIANO, a cross-dispersed NIR spectrograph for the Telescopio Nazionale Galileo (TNG) at the Roque de Los Muchachos Observatory in La Palma (Spain). GIANO delivers a spectrum that covers in a single exposure the wavelength range from $0.95 \mu \mathrm{m}$ to $2.4 \mu \mathrm{m}$ at a resolving power $R \simeq 50000$. The main disperser is a commercial R2 echelle grating with 23.2 lines/mm working in quasi-Littrow configuration on a $d=100 \mathrm{~mm}$ collimated beam. Cross dispersion is achieved via a network of fused silica and ZnSe prisms that work in double pass, that is, they cross-disperse the light both before and after it is dispersed by the echelle gratings, thus producing curved spectral orders. The echellogram on the $2 k \times 2 k$ detector

* Table 3 is available in electronic form at http://www . aanda.org spans 49 orders, from \#32 to \#80. The spectral coverage is complete up to $1.7 \mu \mathrm{m}$. At longer wavelengths the orders become larger than the detector. The effective spectral coverage in the $K$-band is about $75 \%$. Light from the telescope feeds a bundle of two IR-transmitting ZBLAN fibers, with a core of $85 \mu \mathrm{m}$, corresponding to a sky-projected angle of 1 arcsec. The two fibers are aligned and mounted inside a custom connector. The cores are at a distance of $0.25 \mathrm{~mm}$, equivalent to a sky-projected angle of about 3 arcsec.

Owing to the constraints set by the visitor focus, the fiber entrance was coupled to the TNG using a provisional, simplified focal adapter that consisted of a commercial $\mathrm{CaF}_{2}$ singlet lens positioned $26 \mathrm{~mm}$ before the fibers. The focal adapter was mechanically mounted at a fiducial position, no further adjustment of the optical axis was possible. This unfortunately resulted in a very reduced efficiency of the system, and only bright targets were observable at that time. More technical details on the instrument can be found in Oliva et al. (2012a,b, 2013).

As a first science verification of the GIANO performances, we observed three bright red supergiants (RSGs) in the star cluster RSGC2. RSGC2 is one of the few young, massive $(4 \times$ $10^{4} M_{\odot}$ ) clusters in the Galactic plane, located at the base of 
Table 1. RSG stars observed with GIANO.

\begin{tabular}{|c|c|c|c|c|c|c|c|c|c|c|}
\hline Ref & D09 Ref & ID & RA (J2000) & $\operatorname{Dec}(\mathrm{J} 2000)$ & SpT & $J$ & $H$ & $K$ & $A_{K}$ & $R V\left(\mathrm{~km} \mathrm{~s}^{-1}\right)$ \\
\hline$\# 1$ & \#6 & J18391838-0600383 & 183918.386 & -060038.39 & M5I & 7.717 & 5.919 & 5.072 & 1.17 & 107.1 \\
\hline \#2 & \#2 & J18391961-0600408 & 183919.616 & -060040.83 & M3I & 6.899 & 5.045 & 4.120 & 1.39 & 111.1 \\
\hline \#3 & \#3 & J18392461-0602138 & 183924.611 & -060213.80 & M4I & 7.273 & 5.458 & 4.499 & 1.34 & 110.5 \\
\hline
\end{tabular}

Notes. Identification names, coordinates, and magnitudes are taken from 2MASS, reddening $A_{K}$ and radial velocities are taken from Davies et al. (2007).
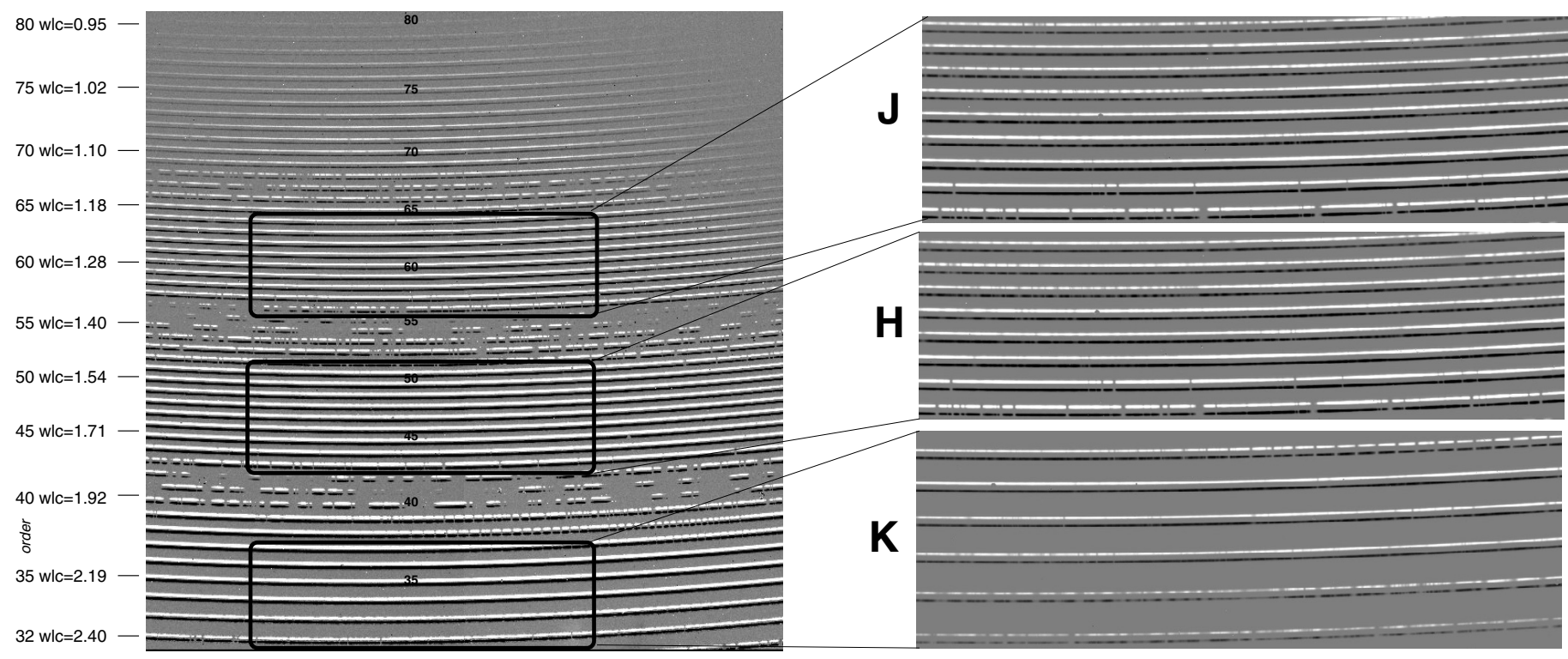

Fig. 1. GIANO 2D (sky-subtracted) spectra of one of the observed RSG stars. Sky subtraction has been performed by nodding on fiber, resulting in one positive and one negative spectrum.

the Scutum-Crux arm and at the tip of the Galactic bar (Davies et al. 2007) at a distance of $\simeq 3.5 \mathrm{kpc}$ from the Galactic center. Such rare clusters are rich in RSG stars and represent ideal laboratories for studying the evolution of massive stars as well as for physically and chemically characterizing the most recent and violent star formation events in the inner Galaxy. However, one can only study them in the IR or at radio wavelengths, because of high visual extinction $\left(A_{V} \approx 10-30 \mathrm{mag}\right)$ that affects the Galactic plane. Chemical abundances of Fe, C, and alpha-elements have been measured in 12 RSG stars, members of RSGC2, by using medium-resolution $(R \simeq 17000)$ spectra obtained with NIRSPEC at the KeckII Telescope (Davies et al. 2009b, hereafter D09). In some of these RSGs Verheyen et al. (2012) also found $\mathrm{SiO}$ maser emission.

We present and discuss the main atomic and molecular lines identified in the GIANO spectra of the observed three RSGs, which are members of the RSGC2 star cluster and are in common with the D09 sample. We also report the inferred chemical abundances of CNO and F from molecular lines, iron-peak, alpha, and other light elements and a few s-process elements from atomic lines.

\section{Observations and data reduction}

For the observed targets, the 2MASS reference name, coordinates, spectral type and magnitudes, reddening and radial velocities from Davies et al. (2007) are reported in Table 1.

The reddening estimates do not affect the line equivalent width measurements since at NIR wavelengths the continuum is still dominated by the stellar photosphere and reddening dilutes both the line and the adjacent continuum flux by the same amount.

The data were collected during the technical night on July 30, 2012. A first exposure of 5 min was acquired by centering the star+sky on fiber \#1 and the sky alone on fiber \#2. Then we acquired a second exposure with the same integration time but with star+sky on fiber \#2 and the sky alone on fiber \#1 by nodding the telescope. By subtracting the $2 \mathrm{D}$ spectrum of the second exposure from the 2D spectrum of the first exposure we obtained the pure star spectrum (see Fig. 1). Nodding on fiber is very effective to properly subtract sky emission and instrumental background. The geometry of the orders was determined using flat exposures with a tungsten calibration lamp. The 2D spectrum was thus rectified and the spectra from each order were extracted by summing six pixels around each fiber, in the direction perpendicular to dispersion. Wavelength calibration was determined by feeding the fibers with the light from a U-Ne lamp. The wavelengths of the uranium lines were taken from Redman et al. (2011), while for neon we used the table available on the NIST (Kramida et al. 2012). The $\lambda$ versus pixel relationship was obtained starting from a physical model of the instrument. This procedure is part of the pipeline that we are developing for the instrument. The resulting wavelength accuracy was about $\lambda / 300000 \mathrm{rms}$, that is, $0.05 \AA$ for lines in the $H$-band, while the overall signal-to-noise ratio is about 50 . For the three observed stars we found radial velocities consistent with the values reported by Davies et al. (2007) and listed in Table 1.

Figure 2 shows a few examples of the observed spectra for iron-peak and s-process elements, Fig. 3 for alpha elements and Fig. 4 for other light elements. 


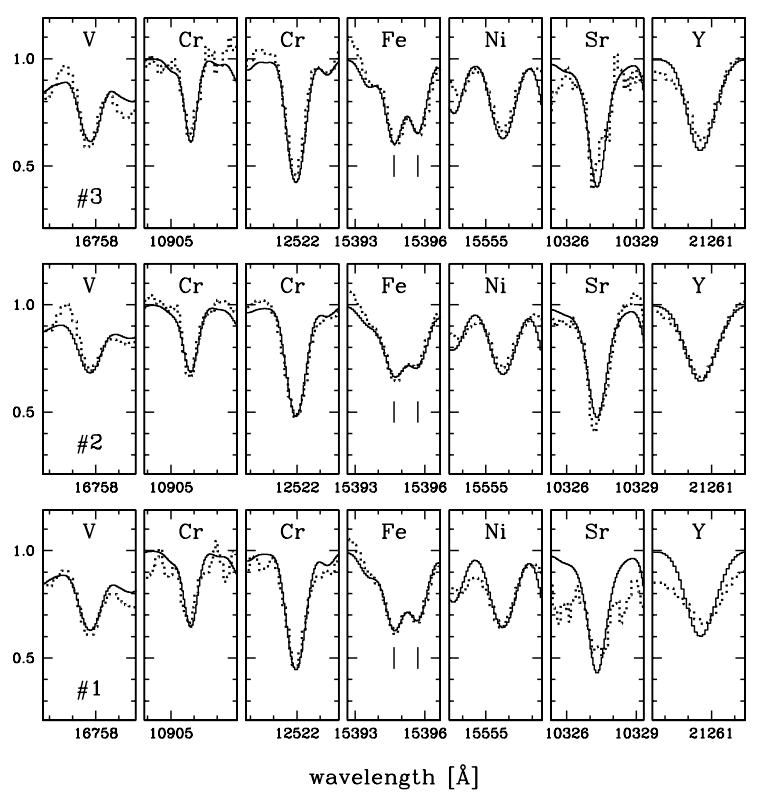

Fig. 2. GIANO spectra around some iron-peak and s-process element lines for the three observed RSG stars (dotted lines). Our best-fit models are overplotted as solid lines. Rest frame wavelengths are defined in air and thickmarks are every $\AA$ in each panel.
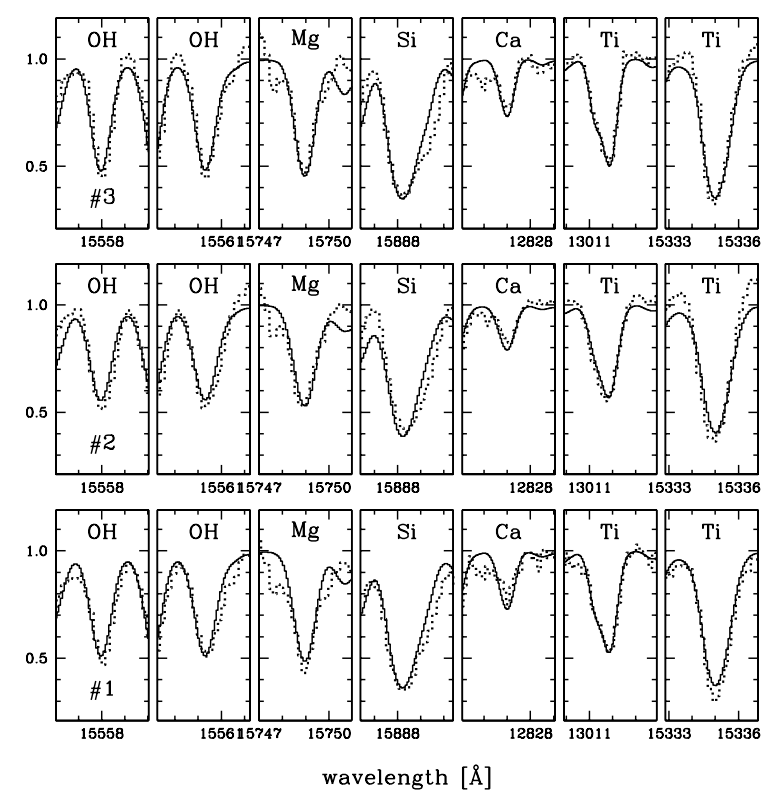

Fig. 3. GIANO spectra around some alpha element lines for the three observed RSG stars (dotted lines). Our best-fit models are overplotted as solid lines. Rest frame wavelengths are defined in air and thickmarks are every $\AA$ in each panel.

\section{Chemical abundance analysis}

Deriving chemical abundances of RSG stars is an intrinsically difficult task for several reasons, namely the high level of molecular blending and blanketing in their spectra, the degeneracy in the determination of surface parameters, and the various broadening effects due to micro and macro turbulence. The use of high-resolution spectroscopy in the NIR significantly mitigates the problem of the molecular blending and blanketing, which remains critical at optical wavelengths.

To measure chemical abundances from the GIANO spectra, we used full spectral synthesis techniques and equivalent width
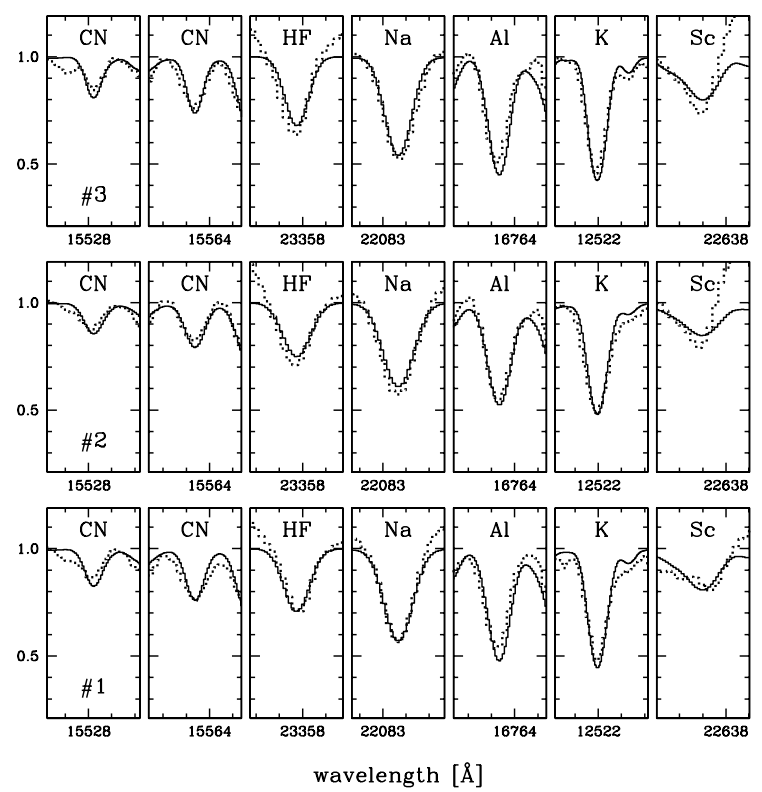

Fig. 4. GIANO spectra around some light element lines for the three observed RSG stars (dotted lines). Our best-fit models are overplotted as solid lines. Rest frame wavelengths are defined in air and thickmarks are every $\AA$ in each panel.

measurements of selected lines, sufficiently isolated, free from significant blending and/or contamination by telluric absorption and without strong wings. The presence of possible telluric absorption was carefully checked on an almost featureless O-star (Hip89584) spectrum.

We computed a large grid of suitable synthetic spectra to model RSG stars by varying the stellar parameters and the element abundances. We used the same code as in D09 to facilitate the comparison. This is an updated version (Origlia et al. 2002) of the program first described in Origlia et al. (1993).

The code was also successfully used to obtain abundances of bulge field (Rich et al. 2012, and references therein) and globular cluster (Origlia et al. 2008, and references therein) giants, young clusters dominated by RSGs (Larsen et al. 2006, 2008), and to study the chemical abundances of RSGs in the Galactic center (Davies et al. 2009a). The code uses the LTE approximation, is based on the molecular blanketed model atmospheres of Johnson et al. (1980) at temperatures $\leq 4000 \mathrm{~K}$ and on the ATLAS 9 models for temperatures above $4000 \mathrm{~K}$, and it includes thousands of NIR atomic transitions from the Kurucz database ${ }^{1}$, Biemont \& Grevesse (1973), and Melendez \& Barbuy (1999), while molecular data are taken from our (Origlia et al. 1993, and subsequent updates) and Plez (priv. comm.) compilations. The reference solar abundances are taken from Grevesse \& Sauval (1998).

Equivalent width measurements of the $\mathrm{OH}$ and $\mathrm{CN}$ molecular lines in the $H$-band, and of one HF line in the $K$-band were used to determine the oxygen, nitrogen and fluorine abundances.

${ }^{12} \mathrm{C}$ and ${ }^{13} \mathrm{C}$ carbon abundances were mostly determined from the $\mathrm{CO}$ bandheads in the $H$ and $K$-bands, respectively, by means of full spectral synthesis, because of the high level of crowding and blending of the $\mathrm{CO}$ lines in these stars. Figure 5 shows the GIANO spectra of the three RSGs centered on some of the $\mathrm{CO}$ bandheads used for determining the carbon abundances and our best-fit models.

\footnotetext{
1 http://www.cfa.harvard.edu/amp/ampdata/kurucz23/ sekur.html
} 

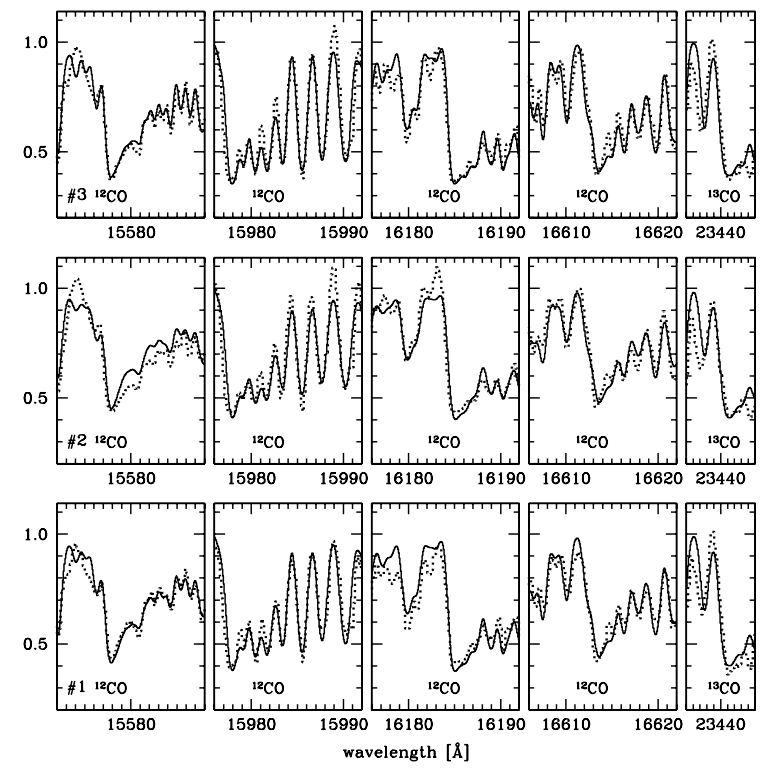

Fig. 5. GIANO $H$-band spectra of the ${ }^{12} \mathrm{CO}(3-0),(5-2),(6-3)$ and (8-5) bandheads and $K$-band spectra of the ${ }^{13} \mathrm{CO}(3-0)$ bandhead for the three observed RSG stars (dotted lines). Our best-fit models are overplotted as solid lines. Rest frame wavelengths are defined in air.

Equivalent width measurements of neutral atomic lines over the full spectral range covered by the GIANO spectra were used to derive abundances of $\mathrm{Fe}$ and other iron-peak elements such as $\mathrm{V}, \mathrm{Cr}, \mathrm{Ni}$, of alpha $(\mathrm{Mg}, \mathrm{Si}, \mathrm{Ca}$ and $\mathrm{Ti}$ ) and other light elements such as $\mathrm{Na}, \mathrm{Al}, \mathrm{K}, \mathrm{Sc}$, and of the s-process element $\mathrm{Y}$, while $\mathrm{Sr}$ abundances were obtained from ionized lines. Table 3 (available online) reports the list of lines used in the present analysis and their measured equivalent widths.

Some other atomic lines of S ( $Y$-band), Mn ( $J$-band), Co, and $\mathrm{Cu}(H$-band) are present in the spectra of the observed RSGs, but they are either too saturated or blended and/or contaminated by telluric absorption, which makes them ineffective for deriving reliable abundances.

This compilation is a first census of the usable lines for abundance analysis and it does not pretend to be complete. Work is in progress to identify other potential lines of interest that, however, will be checked on forthcoming observed spectra of less extreme stars for a proper calibration. We note that our compilation contains several lines in common with the Smith et al. (2013) APOGEE spectral line list.

We compared the observed spectra with models in which temperature, gravity, and microturbulence velocity are as carefully determined in D09, that is $T_{\text {eff }}=3600 \mathrm{~K}, \log g=0.0$ and $\xi=2 \mathrm{~km} \mathrm{~s}^{-1}$ for all the three stars. These parameters were fine-tuned to enable simultaneous spectral fitting of the $\mathrm{CO}$ and $\mathrm{OH}$ molecular bands and the few atomic lines in the NIRSPEC spectra. The many more $\mathrm{CO}, \mathrm{OH}$, and $\mathrm{CN}$ molecualr lines as well as neutral atomic lines available in the GIANO spectra allowed us to carefully verify the reliability of the parameters adopted in D09. Models with these atmospheric parameters satisfactorily reproduce both the D09 and the current GIANO spectra. However, while D09 obtained good fits to the data without including an additional macroturbulence velocity broadening, since this was not resolved at their spectral resolution of $R \simeq 17000$, this is not the case for the GIANO spectra at $R \simeq 50000$. We obtained a good fit to the observed spectra by modeling macroturbulence velocity with a Gaussian $\sigma$ broadening of about $6.4,7.1$, and $5.7 \mathrm{~km} \mathrm{~s}^{-1}$, or equivalently
Table 2. Chemical abundances of the RSG stars observed with GIANO.

\begin{tabular}{|c|c|c|c|c|c|}
\hline \multirow[t]{2}{*}{ Element } & \multirow[t]{2}{*}{ Atomic \# } & \multicolumn{3}{|c|}{$[\mathrm{X} / \mathrm{H}]$} & \multirow[t]{2}{*}{$n_{\text {lines }}$} \\
\hline & & (\#1) & $(\# 2)$ & (\#3) & \\
\hline \multirow[t]{2}{*}{$\mathrm{Fe}$} & \multirow[t]{2}{*}{26} & -0.26 & -0.48 & -0.37 & 13 \\
\hline & & \pm 0.05 & \pm 0.05 & \pm 0.06 & \\
\hline \multirow[t]{2}{*}{ V } & \multirow[t]{2}{*}{23} & -0.36 & -0.43 & -0.30 & 1 \\
\hline & & \pm 0.10 & \pm 0.10 & \pm 0.10 & \\
\hline \multirow[t]{2}{*}{$\mathrm{Cr}$} & \multirow[t]{2}{*}{24} & -0.25 & -0.32 & -0.15 & 4 \\
\hline & & \pm 0.11 & \pm 0.14 & \pm 0.10 & \\
\hline \multirow[t]{2}{*}{$\mathrm{Ni}$} & \multirow[t]{2}{*}{28} & -0.30 & -0.47 & -0.34 & 6 \\
\hline & & \pm 0.05 & \pm 0.07 & \pm 0.04 & \\
\hline \multirow[t]{2}{*}{$\mathrm{Mg}$} & \multirow[t]{2}{*}{12} & -0.19 & -0.60 & -0.33 & 5 \\
\hline & & \pm 0.13 & \pm 0.10 & \pm 0.08 & \\
\hline \multirow[t]{2}{*}{$\mathrm{Si}$} & \multirow[t]{2}{*}{14} & -0.12 & -0.61 & -0.40 & 7 \\
\hline & & \pm 0.03 & \pm 0.07 & \pm 0.05 & \\
\hline \multirow[t]{2}{*}{$\mathrm{Ca}$} & \multirow[t]{2}{*}{20} & -0.29 & -0.48 & -0.40 & 7 \\
\hline & & \pm 0.08 & \pm 0.07 & \pm 0.08 & \\
\hline \multirow[t]{2}{*}{$\mathrm{Ti}$} & \multirow[t]{2}{*}{22} & -0.19 & -0.35 & -0.28 & 21 \\
\hline & & \pm 0.07 & \pm 0.07 & \pm 0.06 & \\
\hline \multirow[t]{2}{*}{$\mathrm{C}$} & \multirow[t]{2}{*}{6} & -0.75 & -0.85 & -0.65 & - \\
\hline & & \pm 0.05 & \pm 0.05 & \pm 0.05 & \\
\hline \multirow[t]{2}{*}{$\mathrm{N}$} & \multirow[t]{2}{*}{7} & +0.23 & +0.01 & +0.07 & 18 \\
\hline & & \pm 0.04 & \pm 0.04 & \pm 0.03 & \\
\hline \multirow[t]{2}{*}{$\mathrm{O}$} & \multirow[t]{2}{*}{8} & -0.41 & -0.41 & -0.32 & 23 \\
\hline & & \pm 0.04 & \pm 0.06 & \pm 0.05 & \\
\hline $\mathrm{F}$ & 9 & -0.23 & -0.34 & -0.11 & 1 \\
\hline & & \pm 0.10 & \pm 0.10 & \pm 0.10 & \\
\hline $\mathrm{Na}$ & 11 & -0.01 & -0.15 & -0.27 & 2 \\
\hline & & \pm 0.20 & \pm 0.14 & \pm 0.11 & \\
\hline $\mathrm{Al}$ & 13 & -0.32 & -0.48 & -0.35 & 3 \\
\hline & & \pm 0.08 & \pm 0.08 & \pm 0.06 & \\
\hline K & 19 & -0.37 & -0.55 & -0.22 & 3 \\
\hline & & \pm 0.07 & \pm 0.05 & \pm 0.09 & \\
\hline $\mathrm{Sc}$ & 21 & -0.22 & -0.13 & -0.07 & 2 \\
\hline & & \pm 0.09 & \pm 0.09 & \pm 0.06 & \\
\hline $\mathrm{Sr}$ & 38 & - & -0.25 & -0.24 & 2 \\
\hline & & - & \pm 0.06 & \pm 0.19 & \\
\hline Y & 39 & -0.61 & -0.67 & -0.67 & 2 \\
\hline & & \pm 0.10 & \pm 0.12 & \pm 0.13 & \\
\hline${ }^{12} \mathrm{C} /{ }^{13} \mathrm{C}$ & - & 9 & 10 & 11 & - \\
\hline & & \pm 1 & \pm 1 & \pm 1 & \\
\hline
\end{tabular}

Doppler-broadening of 9,10 , and $8 \mathrm{~km} \mathrm{~s}^{-1}$, for stars \#1, \#2 and \#3, respectively. We did not find other appreciable line broadening by stellar rotation.

The final average abundances are quoted in Table 2 and plotted in Fig. 6. The impact of using slightly different assumptions for the stellar parameters on the derived abundances is discussed in Sect. 3.1.

Recently, Davies et al. (2013) discussed the problem of the most appropriate temperature scale for RSGs (see also Levesque et al. 2005), given that temperatures differing by several hundreds degrees can be inferred, using different scales. For the sake of consistency we therefore also explore models with significantly warmer $\left(T_{\text {eff }}>4000 \mathrm{~K}\right)$ and cooler $\left(T_{\text {eff }}<3200 \mathrm{~K}\right)$ temperatures than the one adopted in this analysis as best-fit value. We found that for temperatures above $3800 \mathrm{~K}$ we were still able to fit the observed spectra by using very peculiar enhanced $\mathrm{N}$ and $\mathrm{O}$ abundances, providing unlikely $[\mathrm{O} / \mathrm{Fe}]>0.9$ dex and $[\mathrm{C}+\mathrm{N} / \mathrm{Fe}]>1.0$ dex. For temperatures below $3200 \mathrm{~K}$, we were barely (at $>1.5$ sigma level) able to fit the spectra with very peculiar depleted $\mathrm{N}$ and $\mathrm{O}$ abundances, providing $[\mathrm{O} / \mathrm{Fe}]<$ -0.3 dex and $[\mathrm{C}+\mathrm{N} / \mathrm{Fe}] \approx-0.5 \mathrm{dex}$. The impact on the overall iron and iron-peak elemental abundances is much weaker (within 0.1-0.2 dex). 


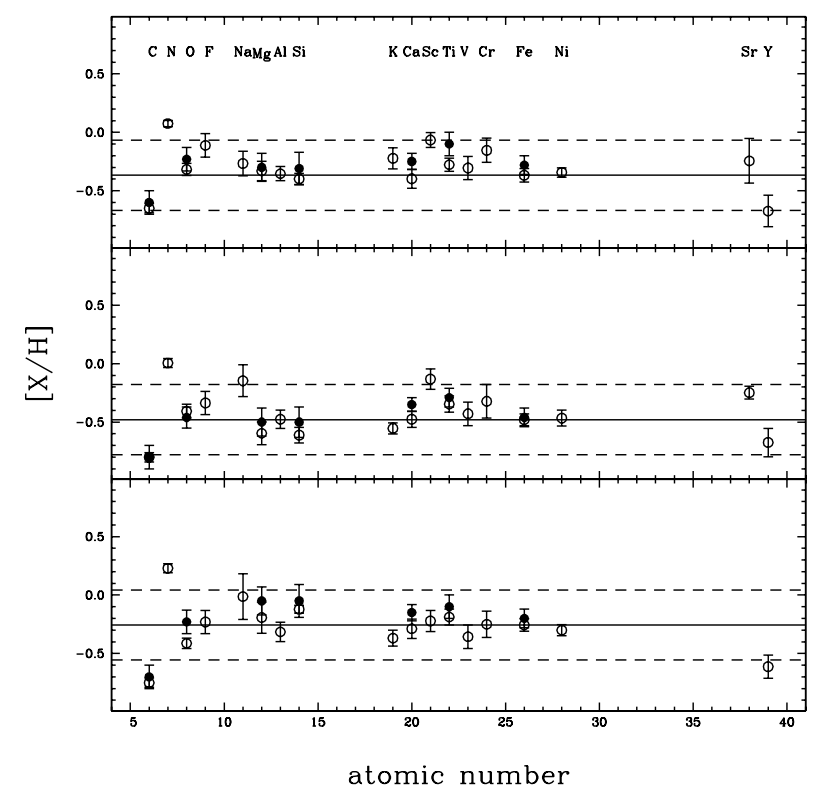

Fig. 6. Open circles: $[\mathrm{X} / \mathrm{H}]$ chemical abundances as a function of the atomic number for the three RSGs observed with GIANO. Solid dots: corresponding $[\mathrm{X} / \mathrm{H}]$ abundances from D09. The horizontal lines mark the $[\mathrm{Fe} / \mathrm{H}]$ abundance (solid line) and the \pm 0.3 dex values (dashed lines), as inferred in the present analysis.

\subsection{Error budget}

We can quantify random and systematic errors in the measurements of the equivalent widths and in the derived chemical abundances as follows: the typical random error of the measured line equivalent widths is between 10 and $20 \mathrm{~m} \AA$, mostly arising from a $\pm 2 \%$ uncertainty in the placement of the pseudo-continuum, as estimated by overlapping the synthetic and the observed spectra. These random uncertainties in the line equivalent width measurements correspond to abundance variations ranging from a few hundredths to 1 tenth of a dex. This $\leq 0.1$ dex error is lower than the typical $1 \sigma$ scatter in the derived abundances from different lines, which normally ranges between 0.1 and 0.2 dex. The errors quoted in Table 2 for the final abundances were obtained by dividing these $1 \sigma$ errors by the squared root of the number of used lines, ranging from a few to about 20. A $10-20 \mathrm{~m} \AA$ variation of line equivalent width value was also obtained by varying the Gaussian line broadening (due to macro turbulence) by $\simeq 1 \mathrm{~km} \mathrm{~s}^{-1}$, but this is mostly a systematic effect.

The other systematics arise from varying the adopted stellar parameters. To properly quantify them, we generated a grid of test models with $T_{\text {eff }}$ between $3400 \mathrm{~K}$ and $3800 \mathrm{~K}, \log g$ between 0.0 and 1.0 dex, and $\xi$ between 2 and $4 \mathrm{~km} \mathrm{~s}^{-1}$.

We found that variations of $\pm 100 \mathrm{~K}$ with respect to the adopted temperature of $3600 \mathrm{~K}$ have a weak effect on the measured equivalent widths of atomic lines (on average the variation is $<10 \mathrm{~m} \AA$ corresponding to a variation of few hundredths dex of the element abundance). Molecular $\mathrm{OH}$ and $\mathrm{CN}$ lines are more sensitive to temperature variations: their equivalent widths can vary by $15-20 \mathrm{~m} \AA$ by varying temperature of $\pm 100 \mathrm{~K}$, corresponding to $\leq 0.1$ dex variation of the element abundance. A variation of $\log g$ by \pm 0.5 dex has a negligible impact on the equivalent width of the $\mathrm{OH}$ lines, while it produces a variation of $\simeq 20 \mathrm{~m} \AA(\simeq 0.15$ dex in abundance $)$ and $\simeq 30 \mathrm{~m} \AA(\simeq 0.20$ dex in abundance) in the equivalent widths of the atomic and $\mathrm{CN}$ lines, respectively. A variation of $\xi$ by $\pm 0.5 \mathrm{~km} \mathrm{~s}^{-1}$ has a negligible impact on the equivalent widths of $\mathrm{CN}$ lines, while it produces a variation of $\simeq 15-20 \mathrm{~m} \AA$ in the equivalent widths of atomic and $\mathrm{OH}$ lines, corresponding to a variation of $\leq 0.1$ dex of the element abundance. A somewhat conservative estimate of the overall systematic uncertainty in the abundance $(A)$ determination, caused by variations of the atmospheric parameters, can be computed as follows: $(\Delta A)^{2}=(\partial A / \partial T)^{2}(\Delta T)^{2}+(\partial A / \partial \log g)^{2}(\Delta \log g)^{2}+$ $(\partial A / \partial \xi)^{2}(\Delta \xi)^{2}$ and it amounts to $0.15-0.20$ dex.

We also determined the statistical significance of our bestfit solution for the spectral synthesis of the $\mathrm{CO}$ features and the derived carbon abundances. As a figure of merit of the statistical test we adopted the difference between the model and the observed spectrum. To quantify systematic discrepancies, this parameter is more powerful than the classical $\chi^{2}$ test, which is instead equally sensitive to random and systematic scatters (see Origlia \& Rich 2004, for more discussion and references).

Our best-fit solutions always show $>90 \%$ probability to be representative of the observed spectra, while those with \pm 0.1 dex carbon abundance are significant at $\geq 1.5 \sigma$ level only.

We also computed other test models with $\Delta T_{\mathrm{eff}}= \pm 200 \mathrm{~K}$, $\Delta \log g= \pm 0.5 \mathrm{dex}$ and $\Delta \xi= \pm 0.5 \mathrm{~km} \mathrm{~s}^{-1}$, and also with corresponding simultaneous variations of the $\mathrm{C}$ abundance (0.1-0.2 dex) to reproduce the depth of the molecular features. $\mathrm{CO}$ molecular bands are very sensitive thermometers in the range of temperature between $4500 \mathrm{~K}$ and $3800 \mathrm{~K}$. Indeed, temperature sets the fraction of molecular versus atomic carbon. At temperatures below $3800 \mathrm{~K}$ most of the carbon is in molecular form, which drastically reduces the dependence of the $\mathrm{CO}$ band strengths on the temperature itself. At temperatures $\geq 4500 \mathrm{~K}$ molecules barely survive, most of the carbon is in atomic form, and the CO spectral features become very weak. Solutions with $\Delta \log g= \pm 0.5$ dex or with $\Delta \xi= \pm 0.5 \mathrm{~km} \mathrm{~s}^{-1}$ and corresponding $0.1-0.2$ dex variation of the carbon abundance are significant at $\geq 1.5 \sigma$ level only.

\section{Results and discussion}

The abundances of all chemical elements with measurable lines in the GIANO spectra are very similar in all the three RSGs, as expected because they are members of a star cluster.

We found iron abundances between half and one third solar, in good agreement with the previous estimates by D09. The other iron-peak elements $(\mathrm{V}, \mathrm{Cr}, \mathrm{Ni})$ show abundances that are consistent with the iron values to within $\pm 0.15 \mathrm{dex}$, thus fully confirming the sub-solar metallicity of the cluster.

The alpha $(\mathrm{O}, \mathrm{Mg}, \mathrm{Si}, \mathrm{Ca}, \mathrm{Ti})$ as well as most of the other light (F, Na, Al,K, Sc) elements behave in a similar fashion, with $[\mathrm{X} / \mathrm{Fe}]$ abundance ratios about solar $( \pm 0.15 \mathrm{dex})$; the only exceptions are $[\mathrm{F} / \mathrm{Fe}]$ and $[\mathrm{Sc} / \mathrm{Fe}]$ in star \#1, $[\mathrm{Na} / \mathrm{Fe}]$ in star \#2 and $[\mathrm{Na} / \mathrm{Fe}]$ and $[\mathrm{Sc} / \mathrm{Fe}]$ in star \#3, which are slightly enhanced (but always within a factor of two).

Interestingly, the measured $[\mathrm{F} / \mathrm{O}]$ abundance ratios $(+0.18$ in star \#1, +0.07 in star \#2 and +0.21 in star \#3), are fully consistent with the values measured in K-M dwarfs in the Orion Nebula Cluster by Cunha \& Smith (2005) and are representative of the disk composition.

The Sr and Y s-process elements behave somewhat differently from each other: $[\mathrm{Sr} / \mathrm{Fe}]$ is slightly enhanced, while $[\mathrm{Y} / \mathrm{Fe}]$ is slightly depleted (by a factor of $\leq 2$ ) with respect to solar.

$[\mathrm{C} / \mathrm{Fe}]$ is depleted by a factor between two and three, similarly to what was found by $\mathrm{D} 09$, and the ${ }^{12} \mathrm{C} /{ }^{13} \mathrm{C}$ ratio is also rather low (between 9 and 11), indicating that some extra-mixing processes in the stellar interiors are at work during the postmain-sequence evolution. Evolutionary tracks of massive stars with rotation (e.g. Meynet \& Maeder 2000) can account for both 
the overall depletion of carbon and the low ${ }^{12} \mathrm{C} /{ }^{13} \mathrm{C}$ isotopic ratio, and indeed, deep, rotationally enhanced mixing was previously suggested by D09.

$[\mathrm{N} / \mathrm{Fe}]$ is enhanced in such a way that $[\mathrm{C}+\mathrm{N} / \mathrm{Fe}]$ is about 0.0 $(+0.00$ in star \#1, +0.12 in star \#2, and +0.16 in star \#3), consistent with standard $\mathrm{CN}$ nucleosynthesis.

The mostly solar-scaled $[\mathrm{X} / \mathrm{Fe}]$ abundance patterns of ironpeak, alpha, and other light elements as measured in the observed three RSGs of RSCG2 are fully consistent with the chemistry of the thin disk (see e.g. Reddy et al. 2003), that underwent chemical enrichment over long timescales with the contribution of both type II and type I supernovae. At variance, the sub-solar metallicity of RSGC2 and of its companion cluster RSGC1 (see D09) is intriguing. Indeed, abundance measurements of Cepheids in the inner disk (see e.g. Genovali et al. 2013; Andrievsky et al. 2013, and references therein) indicate the existence of a positive metallicity gradient toward the inner regions and metal abundances well in excess of solar. However, these measurements still sample a region at a minimum Galactocentric distance of about $4 \mathrm{kpc}$ because of the huge extinction closer to the center. Only a few IR measurements of RSGs exist so far in the innermost disk region and in the Galactic center (e.g. Ramirez et al. 2000; Martins et al. 2008; Najarro et al. 2009; Davies et al. 2009a,b), consistent with a metal abundance of about solar and some level of alpha-element enhancement (see e.g. Cunha et al. 2007).

It is therefore clear that neither RSGs in the Scutum star clusters (D09 and the present work) nor RSGs in the center of the Galaxy follow the radial Galactic trend traced by Cepheids and other young stellar populations at Galactocentric distances RGC > 3-4 kpc (see D09 and Genovali et al. 2013, for more discussion and references). However, this is not surprising, because the innermost region of the Galaxy shows several different substructures, such as the bulge/bar and the ends of the spiral arms, with strong fluctuations in stellar/gas density and star formation rates. In such a complex physical and kinematic environment, the chemical enrichment process is also expected to have been quite inhomogeneous.

\section{Conclusions}

As a preliminary test of the scientific performances of the GIANO spectrograph during its first commissioning in July 2012, we observed three bright RSGs in the Scutum star cluster RSGC2.

A NIR spectrograph such as GIANO, which provides high spectral resolution and full spectral coverage in a single exposure, is a unique instrument to perform detailed chemical studies of cool stars in any Galactic environment, including highly reddened star clusters such as RSGC2 in the inner Galactic disk.

The high spectral resolution has allowed us to resolve and measure the macro turbulence broadening in the observed RSGs and to better identify unblended lines for accurate equivalent width measurements and chemical abundance determinations.

The simultaneous access to the $Y J H$ and $K$-bands offers the possibility of sampling most of the elements of interest for a complete check of the chemical clock and of measuring from a few to several tens of lines per element, thus enabling an accurate and statistically significant abundance analysis. We used the $Y$-band to measure $\mathrm{Cr}$ and $\mathrm{Sr}$, the $J$-band to measure $\mathrm{K}$ and some $\mathrm{Fe}, \mathrm{Cr}, \mathrm{Ca}, \mathrm{Mg}, \mathrm{Si}$, and Ti lines, the $H$-band to measure ${ }^{12} \mathrm{C}, \mathrm{N}$, $\mathrm{O}, \mathrm{Al}, \mathrm{V}$, and $\mathrm{Ni}$, most of the $\mathrm{Fe}$ lines and some $\mathrm{Al} \mathrm{Ca}, \mathrm{Mg}, \mathrm{Si}$ and Ti lines, and finally the $K$-band to measure ${ }^{13} \mathrm{C}, \mathrm{F}, \mathrm{Na}, \mathrm{Sc}, \mathrm{Y}$ and a few $\mathrm{Al}, \mathrm{Ti}$ and $\mathrm{Fe}$ lines.

For the three observed RSGs we found overall abundance patterns consistent with the thin-disk chemistry, and we confirmed the sub-solar metallicity of the RSGC2 star cluster, as previously suggested by D09, which indicates that the inner disk in particular, but more generally the inner Galaxy, has quite an inhomogeneous chemical composition.

Acknowledgements. Part of this work was supported by the grant TECNOINAF-2011. This publication makes use of data products from the Two Micron All Sky Survey, which is a joint project of the University of Massachusetts and the Infrared Processing and Analysis Center/California Institute of Technology, funded by the National Aeronautics and Space Administration and the National Science Foundation. We thank Ben Davies for his carefull referee report.

\section{References}

Andrievsky, S. M., Lepine, J. R. D., Korotin, S. A., et al. 2013, MNRAS, 428, 3252

Biemont, E., \& Grevesse, N. 1973, Atomic Data and Nuclear Data Tables, 12 221

Davies, B., Figer, D. F., Kudritzki, R., et al. 2007, ApJ, 671, 78

Davies, B., Origlia, L., Kudritzki, R., et al. 2009a, ApJ, 694, 46

Davies, B., Origlia, L., Kudritzki, R., et al. 2009b, ApJ, 696, 2014

Davies, B., Kudritzki, R., Plez, B., et al. 2013, ApJ, 767, 3

Cunha, K., \& Smith, V. V. 2005, ApJ, 626, 425

Cunha, K., Sellgren, K., Smith, V. V., et al. 2007, ApJ, 669, 1011

Genovali, K., Lemasle, B., Bono, G., et al. 2013, A\&A, 554, A132

Grevesse, N., \& Sauval, A. J. 1998, Space Sci. Rev., 85, 161

Johnson, H. R., Bernat, A. P., \& Krupp, B. M. 1980, ApJS, 42, 501

Kramida, A., Ralchenko, Yu., Reader, J., \& NIST ASD Team 2012, NIST

Atomic Spectra Database (version 5.0), http://physics.nist.gov/asd

Larsen, S. S., Origlia, L., Brodie, J., \& Gallagher, J. S. 2006, MNRAS, 368, 10

Larsen, S. S., Origlia, L., Brodie, J., \& Gallagher, J. S. 2008, MNRAS, 383 263

Levesque, E. M., Massey, P., Olsen, K. A. G., et al. 2005, ApJ, 628, 973

Martins, F., Hillier, D. J., Paumard, T., et al. 2008, A\&A, 478, 219

Meynet, G., \& Maeder, A. 2000, A\&A, 361, 101

Melendez, J., \& Barbuy, B. 1999, ApJS 124, 527

Najarro, F., Figer, D. F., Hillier, D. J., Geballe, T. R., \& Kudritzki, R. P. 2009, ApJ, 691, 1816

Oliva, E., Origlia, L., Maiolino, R., et al. 2012a, SPIE, 8446, 3

Oliva, E., Biliotti, V., Baffa, C., et al. 2012b, SPIE, 8453, 2

Oliva, E., Origlia, L., Maiolino, R., et al. 2013, A\&A, 555, A78

Origlia, L., \& Rich, R. M. 2004, AJ, 127, 3422

Origlia, L., Moorwood, A. F. M., \& Oliva, E. 1993, A\&A, 280, 536

Origlia, L., Rich, R. M., \& Castro, S. 2002, AJ, 123, 1559

Origlia, L., Valenti, E., \& Rich, R. M. 2008, MNRAS, 388, 1419

Ramirez, S. V., Sellgren, K., Carr, J. S., et al. 2000, ApJ, 537, 205

Reddy, B. E., Tomkin, J., Lambert, D. L., \& Allende Prieto, C. 2003, MNRAS, 340,304

Redman, S. L., Lawler, J. E., Nave, G., Ramsey, L. W., \& Mahadevan, S. 2011, ApJS, 195, 24

Rich, R. M., Origlia, L., \& Valenti, E. 2012, ApJ, 746, 59

Smith, V. V., Cunha, K., Shetrone, M. D., et al. 2013, ApJ, 765, 16

Verheyen, L., Messineo, M., \& Menten K. M. 2012, A\&A, 541, A36

Pages 7 to 8 are available in the electronic edition of the journal at http://www . aanda. org 
L. Origlia et al.: GIANO-TNG spectroscopy of red supergiants in RSGC2

Table 3. Lines identified in the RSG stars observed with GIANO.

\begin{tabular}{|c|c|c|c|c|c|}
\hline $\begin{array}{l}\lambda_{\text {air }} \\
\mu \mathrm{m} \\
\end{array}$ & $\log g f$ & $\begin{array}{c}\chi \\
\mathrm{eV}\end{array}$ & $\begin{array}{c}E W(\# 1) \\
\mathrm{m} \AA\end{array}$ & $\begin{array}{c}E W(\# 2) \\
\mathrm{m} \AA\end{array}$ & $\begin{array}{c}E W(\# 3) \\
m \AA\end{array}$ \\
\hline \multicolumn{6}{|l|}{ Fe I lines } \\
\hline 1.22133 & -1.94 & 4.64 & 133 & 88 & 93 \\
\hline 1.22271 & -1.46 & 4.61 & 160 & 140 & 146 \\
\hline 1.23429 & -1.56 & 4.64 & 186 & 151 & 139 \\
\hline 1.26159 & -1.61 & 4.64 & 131 & 127 & 128 \\
\hline 1.28249 & -4.19 & 3.02 & 193 & 170 & 151 \\
\hline 1.28406 & -1.47 & 4.96 & 134 & 140 & 152 \\
\hline 1.52075 & 0.23 & 5.39 & 378 & 360 & 336 \\
\hline 1.52450 & -0.28 & 5.59 & 349 & 302 & 303 \\
\hline 1.53947 & -0.12 & 5.62 & 360 & 370 & 333 \\
\hline 1.53957 & -0.26 & 5.62 & 296 & 284 & 291 \\
\hline 1.57694 & 0.60 & 5.54 & 469 & 466 & 403 \\
\hline 1.59649 & -0.08 & 5.92 & 268 & 269 & 263 \\
\hline 2.23808 & -0.46 & 5.04 & 447 & 424 & 410 \\
\hline \multicolumn{6}{|l|}{ V I lines } \\
\hline 1.67577 & -1.37 & 3.41 & 296 & 291 & 288 \\
\hline \multicolumn{6}{|l|}{ Cr I lines } \\
\hline 1.06721 & -1.37 & 3.02 & 212 & 189 & 256 \\
\hline 1.08014 & -1.72 & 3.01 & 188 & 195 & 182 \\
\hline 1.09059 & -0.65 & 3.44 & 217 & 248 & 215 \\
\hline 1.25218 & -1.59 & 2.71 & 379 & 383 & 327 \\
\hline \multicolumn{6}{|l|}{$\mathrm{Ni}$ I lines } \\
\hline 1.55554 & 0.07 & 5.49 & 320 & 272 & 271 \\
\hline 1.63105 & 0.07 & 5.29 & 333 & 338 & 299 \\
\hline 1.63642 & 0.44 & 5.29 & 474 & 482 & 454 \\
\hline 1.65893 & -0.49 & 5.47 & 156 & 159 & 161 \\
\hline 1.69967 & 0.31 & 5.31 & 339 & 337 & 295 \\
\hline 1.70016 & 0.23 & 5.49 & 250 & 217 & 219 \\
\hline \multicolumn{6}{|c|}{ Mg I lines } \\
\hline 1.18282 & -0.29 & 4.35 & 392 & 402 & 377 \\
\hline 1.57407 & -0.24 & 5.94 & 419 & 382 & 374 \\
\hline 1.57490 & -0.06 & 5.94 & 486 & 452 & 405 \\
\hline 1.57658 & 0.38 & 5.94 & 490 & 482 & 434 \\
\hline 1.59545 & -1.03 & 6.59 & 287 & 239 & 249 \\
\hline \multicolumn{6}{|l|}{ Si I lines } \\
\hline 1.19916 & -0.16 & 4.92 & 308 & 258 & 241 \\
\hline 1.21035 & -0.39 & 4.93 & 283 & 278 & 247 \\
\hline 1.22707 & -0.41 & 4.96 & 279 & 248 & 240 \\
\hline 1.58884 & -0.03 & 5.09 & 554 & 567 & 493 \\
\hline 1.59601 & 0.13 & 5.99 & 417 & 386 & 370 \\
\hline 1.60600 & -0.44 & 5.96 & 311 & 239 & 285 \\
\hline 1.60948 & -0.11 & 5.97 & 371 & 315 & 324 \\
\hline \multicolumn{6}{|l|}{ Ca I lines } \\
\hline 1.28160 & -0.63 & 3.91 & 220 & 222 & 199 \\
\hline 1.28239 & -0.85 & 3.91 & 209 & 221 & 219 \\
\hline 1.28271 & -1.33 & 3.91 & 157 & 124 & 117 \\
\hline 1.61508 & 0.36 & 5.30 & 364 & 354 & 331 \\
\hline 1.61552 & -0.02 & 5.30 & 277 & 211 & 218 \\
\hline 1.61574 & 0.49 & 5.32 & 327 & 287 & 289 \\
\hline 1.61971 & 0.64 & 5.30 & 439 & 406 & 363 \\
\hline \multicolumn{6}{|l|}{ Ti I lines } \\
\hline 1.17805 & -2.38 & 1.44 & 296 & 241 & 224 \\
\hline 1.17972 & -2.46 & 1.43 & 289 & 309 & 273 \\
\hline 1.18929 & -1.91 & 1.43 & 326 & 356 & 305 \\
\hline 1.19495 & -1.76 & 1.44 & 303 & 328 & 279 \\
\hline 1.23884 & -2.20 & 2.16 & 166 & 134 & 135 \\
\hline 1.25696 & -2.26 & 2.18 & 205 & 167 & 208 \\
\hline 1.26711 & -2.52 & 1.43 & 244 & 322 & 304 \\
\hline 1.27384 & -1.39 & 2.18 & 258 & 283 & 262 \\
\hline 1.27449 & -1.35 & 2.49 & 259 & 220 & 190 \\
\hline
\end{tabular}

Notes. Wavelengths are defined in air $\left(\lambda_{\text {air }}=\lambda_{\text {vacuum }} / 1.000274\right)$. We also list the line transition probability $(\log g f)$, excitation potential $(\chi)$ and the measured equivalent widths in the three observed RSG stars.
Table 3. continued.

\begin{tabular}{|c|c|c|c|c|c|}
\hline $\begin{array}{l}\lambda_{\text {air }} \\
\mu \mathrm{m}\end{array}$ & $\log g f$ & $\begin{array}{c}\chi \\
\mathrm{eV}\end{array}$ & $\begin{array}{c}E W(\# 1) \\
\mathrm{m} \AA\end{array}$ & $\begin{array}{c}E W(\# 2) \\
\mathrm{m} \AA\end{array}$ & $\begin{array}{c}E W(\# 3) \\
\mathrm{m} \AA\end{array}$ \\
\hline 1.28217 & -1.53 & 1.46 & 411 & 439 & 360 \\
\hline 1.28314 & -1.11 & 1.43 & 382 & 381 & 349 \\
\hline 1.28470 & -1.55 & 1.44 & 382 & 401 & 355 \\
\hline 1.29199 & -1.22 & 2.15 & 258 & 209 & 226 \\
\hline 1.29876 & -1.78 & 2.51 & 143 & 167 & 161 \\
\hline 1.30054 & -2.46 & 2.18 & 161 & 155 & 173 \\
\hline 1.30119 & -2.49 & 1.44 & 313 & 317 & 280 \\
\hline 1.53348 & -1.15 & 1.89 & 596 & 608 & 512 \\
\hline 1.55438 & -1.48 & 1.88 & 558 & 580 & 497 \\
\hline 2.17829 & -1.16 & 1.75 & 735 & 726 & 648 \\
\hline 2.18974 & -1.45 & 1.74 & 644 & 615 & 595 \\
\hline 2.24439 & -2.25 & 1.74 & 536 & 478 & 486 \\
\hline \multicolumn{6}{|l|}{ Al I lines } \\
\hline 1.67190 & 0.15 & 4.09 & 477 & 478 & 446 \\
\hline 1.67634 & -0.55 & 4.09 & 421 & 423 & 394 \\
\hline 2.10930 & -0.31 & 4.09 & 509 & 503 & 475 \\
\hline \multicolumn{6}{|l|}{$\mathrm{Na} I$ lines } \\
\hline 2.20837 & -0.02 & 3.19 & 538 & 548 & 494 \\
\hline 2.33791 & 0.53 & 3.76 & 566 & 563 & 489 \\
\hline \multicolumn{6}{|l|}{ K I lines } \\
\hline 1.17728 & 0.51 & 1.62 & 293 & 287 & 292 \\
\hline 1.24323 & -0.44 & 1.61 & 222 & 234 & 244 \\
\hline 1.25221 & -0.14 & 1.62 & 358 & 353 & 318 \\
\hline \multicolumn{6}{|l|}{ Sc I lines } \\
\hline 2.17305 & -1.68 & 1.44 & 447 & 513 & 490 \\
\hline 2.26370 & -2.17 & 1.44 & 313 & 367 & 337 \\
\hline \multicolumn{6}{|l|}{ Sr II lines } \\
\hline 1.03273 & -0.35 & 1.84 & - & 387 & 325 \\
\hline 1.09149 & -0.64 & 1.81 & - & 383 & 344 \\
\hline \multicolumn{6}{|l|}{ Y I lines } \\
\hline 2.12604 & -0.10 & 1.43 & 419 & 446 & 417 \\
\hline 2.25438 & -0.20 & 1.40 & - & 362 & 346 \\
\hline \multicolumn{6}{|c|}{${ }^{12} \mathrm{C}^{14} \mathrm{~N}$ lines } \\
\hline 1.52132 & -2.00 & 0.83 & 198 & 194 & 197 \\
\hline 1.52195 & -1.80 & 0.73 & 313 & 287 & 267 \\
\hline 1.52604 & -1.35 & 0.92 & 235 & 195 & 180 \\
\hline 1.52689 & -1.41 & 1.29 & 161 & 123 & 121 \\
\hline 1.52726 & -1.57 & 0.77 & 202 & 174 & 162 \\
\hline 1.52916 & -1.92 & 0.85 & 144 & 162 & 135 \\
\hline 1.53071 & -1.90 & 0.85 & 168 & 166 & 162 \\
\hline 1.53214 & -1.75 & 0.79 & 203 & 183 & 168 \\
\hline 1.53974 & -1.80 & 0.91 & 227 & 211 & 177 \\
\hline 1.54396 & -1.77 & 0.93 & 184 & 193 & 176 \\
\hline 1.54471 & -1.16 & 1.09 & 217 & 214 & 196 \\
\hline 1.54662 & -1.15 & 1.09 & 238 & 230 & 205 \\
\hline 1.55282 & -1.71 & 1.00 & 143 & 117 & 126 \\
\hline 1.55634 & -1.14 & 1.15 & 219 & 139 & 193 \\
\hline 1.56093 & -1.51 & 0.94 & 217 & 199 & 203 \\
\hline 1.56136 & -1.14 & 1.18 & 282 & 225 & 224 \\
\hline 1.56736 & -1.65 & 1.07 & 288 & 207 & 221 \\
\hline 1.56749 & -1.65 & 1.09 & 208 & 164 & 198 \\
\hline \multicolumn{6}{|c|}{${ }^{16} \mathrm{O}^{1} \mathrm{H}$ lines } \\
\hline 1.51458 & -5.63 & 0.16 & 425 & 478 & 427 \\
\hline 1.51479 & -5.63 & 0.16 & 391 & 461 & 400 \\
\hline 1.52366 & -5.93 & 0.45 & 260 & 349 & 238 \\
\hline 1.53285 & -5.67 & 0.47 & 451 & 441 & 378 \\
\hline 1.53912 & -5.59 & 0.49 & 330 & 354 & 325 \\
\hline 1.54092 & -5.55 & 0.26 & 376 & 451 & 401 \\
\hline 1.54698 & -5.26 & 0.94 & 220 & 250 & 309 \\
\hline 1.54969 & -5.26 & 0.91 & 302 & 306 & 267 \\
\hline 1.55053 & -5.46 & 0.52 & 358 & 438 & 383 \\
\hline 1.55580 & -5.49 & 0.30 & 452 & 461 & 417 \\
\hline 1.55602 & -5.49 & 0.30 & 422 & 455 & 418 \\
\hline
\end{tabular}


Table 3. continued.

\begin{tabular}{lccccc}
\hline \hline $\begin{array}{l}\lambda_{\text {air }} \\
\mu \mathrm{m}\end{array}$ & $\log g f$ & $\begin{array}{c}\chi \\
\mathrm{eV}\end{array}$ & $\begin{array}{c}E W(\# 1) \\
\mathrm{m} \AA\end{array}$ & $\begin{array}{c}E W(\# 2) \\
\mathrm{m} \AA\end{array}$ & $\begin{array}{c}E W(\# 3) \\
\mathrm{m} \AA\end{array}$ \\
\hline 1.55658 & -5.42 & 0.90 & 234 & 227 & 228 \\
1.55688 & -5.45 & 0.30 & 395 & 432 & 409 \\
1.55721 & -5.45 & 0.30 & 418 & 463 & 418 \\
1.56519 & -5.29 & 0.53 & 324 & 270 & 273 \\
1.56535 & -5.29 & 0.53 & 389 & 366 & 345 \\
1.58977 & -5.36 & 0.41 & 442 & 465 & 434 \\
1.59104 & -5.14 & 0.60 & 359 & 338 & 357 \\
1.59127 & -5.14 & 0.60 & 370 & 373 & 363 \\
1.63522 & -5.00 & 0.74 & 448 & 442 & 442 \\
1.63546 & -5.00 & 0.74 & 365 & 333 & 353 \\
1.63681 & -4.96 & 0.73 & 359 & 331 & 368 \\
1.68723 & -5.15 & 0.76 & 372 & 353 & 388 \\
\hline${ }^{1} \mathrm{H}^{9} \mathrm{~F}$ lines & & & & & \\
2.33577 & -3.95 & 0.48 & 372 & 353 & 388 \\
\hline
\end{tabular}

